\title{
VESICOSTOMÍA EN LA EDAD PEDIÁTRICA. NUESTRA EXPERIENCIA EN 43 PACIENTES
}

\author{
J.A. QUEIPO ZARAGOZÁ, C. DOMÍNGUEZ HINAREJOS, A. SERRANO DURBÁ, \\ F. ESTORNELL MORAGUES, M. MARTÍNEZ VERDUCH, F. GARCÍA IBARRA
}

\author{
Servicio de Urología Infantil. Hospital Infantil Universitario La Fe de Valencia. Valencia.
}

Actas Urol Esp. 27 (1): 33-38, 2003

\section{RESUMEN}

"VESICOSTOMÍA EN LA EDAD PEDIÁTRICA. NUESTRA EXPERIENCIA EN 43 PACIENTES"

INTRODUCCIÓN Y OBJETIVOS: La vesicostomía cutánea (VC) es una técnica de derivación urinaria temporal, de elección en pacientes con dilatación del tracto urinario superior (TUS), secundaria a patología vesical o infravesical. El objetivo de este trabajo es mostrar nuestra experiencia en niños sometidos a esta derivación, analizando su eficacia para prevenir la infección urinaria, mejorar o resolver la ureteroectasia y estabilizar o mejorar la función renal; en definitiva la recuperación morfológica y funcional del TUS.

MATERIAL Y MÉTODOS: Presentamos 43 niños, con una edad media de 2,5 años (rango: 1 mes - 14 años) a los que realizamos vesicostomía cutánea (técnica de Blocksom en 36 y el procedimiento de Lapides en el resto) por presentar ureterohidronefrosis grado IV-V, infección urinaria persistente y/o deterioro de la función renal. De ellos, 30 presentaban vejiga neuropática (26 con reflujo vesicoureteral secundario), 7 reflujo vesicoureteral primario masivo y 6 niños válvulas de uretra posterior.

RESULTADOS: El 100\% de los niños con vejiga neurogénica (30 casos), presentaron tras la derivación, mejoría en la ectasia ureteropielocalicial, y el 90\% mejoraron su función renal, desapareciendo el reflujo vesicoureteral en el 65\%. En 12 pacientes se realizó cistoplastia de ampliación con intestino durante el cierre de la vesicostomia, con derivación tipo Mitrofanoff en 5 de ellos. En otros 7 niños se realizó como único procedimiento, la misma derivación continente con apéndice cecal. Los 11 niños restantes mantienen en la actualidad la vesicostomía.

En 5 de los 7 niños con reflujo vesicoureteral primario, éste desapareció, procediéndose al cierre de la vesicostomía. En los otros 2 fue necesario, en el mismo acto quirúrgico, la corrección del reflujo. De los 6 niños afectos de válvulas de uretra posterior, 2 recibieron un trasplante renal, uno se encuentra en lista de espera y el resto (50\%) mantienen una función renal aceptable.

Durante el primer año de seguimiento, 4 pacientes presentaron infección urinaria sintomática, que desapareció posteriormente. El resto, ocasionalmente presentó bacteriuria asintomática que no fue necesario tratar. Como complicaciones se produjo prolapso vesical por la ostomía en 4 pacientes, litiasis en 3 y estenosis del estoma en 2.

CONCLUSIONES: Consideramos que la vesicostomía es la técnica de derivación urinaria electiva en los casos de obstrucción del tramo común y/o ureterohidronefrosis no obstructiva, en neonatos que no mejoran con tratamiento conservador.

PALABRAS CLAVE: Vesicostomía. Vejiga neurógena. Reflujo vesicoureteral. Válvulas de uretra posterior. Ureterohidronefrosis.

\section{ABSTRACT}

"PAEDIATRIC VESICOSTOMY. OUR EXPERIENCE IN 43 PATIENTS”

INTRODUCTION AND OBJECTIVES: Cutaneous vesicostomy (CV) is a choice temporary urinary diversion technique for patients with upper urinary tract (URT) dilation, secondary to vesical o infravesical disease. The objective of this study is to share our experience in children undergoing such diversion, analysing its efficacy to prevent urinary infections, improve or resolve the ureterectasia, and stabilise or improve renal function; in short the functional and morphological recovery of URT.

MATERIAL AND METHODS: 43 children, mean age 2,5 years (range: 1 month - 14 years) with ureterohydronephrosis grade IV-V, persistent urinary infection and/or renal function impairment underwent cutaneous vesicostomy (Blocksom's technique in 36 and Lapides' procedure in the rest). Thirty subjects had neuropathic bladder ( 26 with secondary vesicoureteral reflux), 7 massive primary vesicoureteral reflux, and 6 posterior urethral valve.

RESULTS: All children (100\%) with neurogenic bladder (30 cases), showed improvement of ureteral-pyelic-calyceal ectasia after the diversion, and $90 \%$ improved renal function with disappearance of vesiculoureteral reflux in $65 \%$. Augmentation cystoplasty with gut was performed in 12 patients during vesicostomy closure, using Mitrofanoff's type diversion in 5 of them. The same continent diversion with cecal appendix was used in another 7 children as the only procedure. The remaining 11 children still retain their vesicostomy.

In 5 of the 7 children with primary vesicoureteral reflux, the reflux disappeared and so the vesicostomy was closed. Reflux correction during the same surgical procedure was required for the other 2 children. Of the 6 children with posterior urethral valves, 2 received a renal transplant, one is in waiting list and the rest (50\%) maintain an acceptable renal function.

In the first year of follow-up, 4 patients developed symptomatic urinary infection that later disappeared. The rest had occasional asymptomatic bacteriuria that required no treatment. Complications included vesical prolapse due to ostomy in 4 patients, lithiasis in 3 and stomal stenosis in 2.

CONCLUSIONS: We consider vesicostomy to be the choice urinary diversion technique in cases of common section obstruction and/or nonobstructive ureterohydronephrosis in new-borns who do not improve with conservative treatment.

KEY WORDS: Vesicostomy. Neurogenic bladder. Vesicoureteral reflux. Posterior urethral valves. Ureterohydronephrosis. 


\section{INTRODUCCIÓN Y OBJ ETIVOS}

Las opciones de derivación urinaria transitoria no intubadas en el niño son: la pielostomía, ureterostomía y vesicostomía cutáneas. Esta última, la más utilizada en la actualidad, presenta la ventaja de convertir la vejiga de forma inmediata en un sistema de nula presión, lo que conlleva la descompresión del tracto urinario superior ${ }^{1}$.

Fue referida por primera vez por Blocksom en 1957 y posteriormente modificada por Lapides en 1960 y popularizada por Duckett ${ }^{2}$. Inicialmente se indicó en pacientes con vejiga neurógena (VN), con tan buenos resultados, que hizo que se ampliaran las indicaciones a otras patologías, como el reflujo vesicoureteral (RVU), válvulas de uretra posterior (VUP) y Síndrome de Prune-Belly.

Se realiza en niños no candidatos a una cirugía reconstructora precoz, que supondría mayor morbi-mortalidad y menor probabilidad de recuperación de la función renal, en comparación con la derivación inicial y posterior tratamiento definitivo.

En este trabajo analizamos los resultados en una serie de 43 niños con los diagnósticos de VN, RVU y VUP, sometidos a vesicostomía cutánea, en la mayoría por la técnica de Blocksom modificada.

\section{MATERIAL Y MÉTODOS}

Hemos realizado un estudio retrospectivo sobre una serie de 43 pacientes (25 niños y 18 niñas), sometidos a vesicostomía cutánea en nuestro Servicio, entre los años 1981 y 2001. Las indicaciones para realizar la derivación fueron: alteración de la función renal con dilatación severa del T.U.S. y alteración en el vaciado vesical cuando no era posible el cateterismo intermitente (CI), por problemas mecánicos de uretra o socio-familiares. En el momento de la intervención, la edad media fue de 2,5 años (rango: 1 mes - 14 años), variando en función de la patología de base (Tabla I). La técnica de derivación utilizada ha sido la de Blocksom en 36 niños y la de Lapides en 7.

La patología por la que se realizó dicha derivación queda reflejada en la Tabla I: VN (30/43), RVU primario $(7 / 43)$ y VUP $(6 / 43)$.

\section{RESULTADOS}

De los 30 casos afectos de Vejiga Neurógena, en 25 fue secundaria a mielomeningocele, lipomeningocele en 4 y agenesia sacra en 1 (13 niños y
TABLA I

DISTRIBUCIÓN DE CASOS POR PATOLOGÍA DE BASE/EDAD

\begin{tabular}{|c|c|c|}
\hline & № de casos & $\begin{array}{l}\text { Edad media } \\
\text { (años) }\end{array}$ \\
\hline $\mathbf{V N}$ & 30 & 3 \\
\hline RVUP & 7 & 2,5 \\
\hline VUP & 6 & $\begin{array}{l}1,5^{*} \\
* \text { Meses }\end{array}$ \\
\hline
\end{tabular}

VUP: Válvulas de uretra posterior

17 niñas), y de ellos, 26 presentaban RVU secundario (81\% de incidencia bilateral, 21 de 26). Este RVU fue de alto grado (IV, V) en el $85 \%$ de los casos $(22 / 26)$. El patrón urodinámico predominante fue arreflexia vesical e hipertonía esfinteriana en $63 \%$ $(19 / 26)$, presentando el $37 \%$ restante una vejiga de escasa capacidad e hipertonía vesical.

La necesidad de realizar la derivación vino determinada por la presencia de ureterohidronefrosis (UHN) persistente, pese al cateterismo intermitente (CI), con alteración de la función renal y/o infecciones urinarias recidivantes sintomáticas (éstas últimas presentes en el 83\% de los casos).

La UHN mejoró en el 100\% de los casos, la función renal en el 90\% (27 casos) y el RVU desapareció en el 65\% (17 de 26) (Fig. 1).

$\mathrm{El}$ cierre de la vesicostomía se ha realizado en 19 pacientes, con un tiempo medio de derivación de 4,8 años. En 12 pacientes se realizó cistoplastia de ampliación con intestino en el mismo acto quirúrgico, con Mitrofanoff en 5 de ellos. En los otros 7 niños se realizó como único procedimiento, derivación continente por el mismo principio con apéndice cecal, por dificultades en el sondaje uretral. Once siguen derivados, pendientes de evolución.

En 7 pacientes (6 niños y una niña) la indicación de la derivación fue Reflujo Vesicoureteral Primario, unido a infección urinaria de repetición, a pesar de la profilaxis antibiótica, y/o alteración de la función renal (indicación princeps en dos de ellos). El RVU fue bilateral en todos los casos, presentando 2 pacientes duplicidad pieloureteral unilateral. La edad media en el momento de la derivación fue de 2,5 años (rango: 1 mes-5 años). 

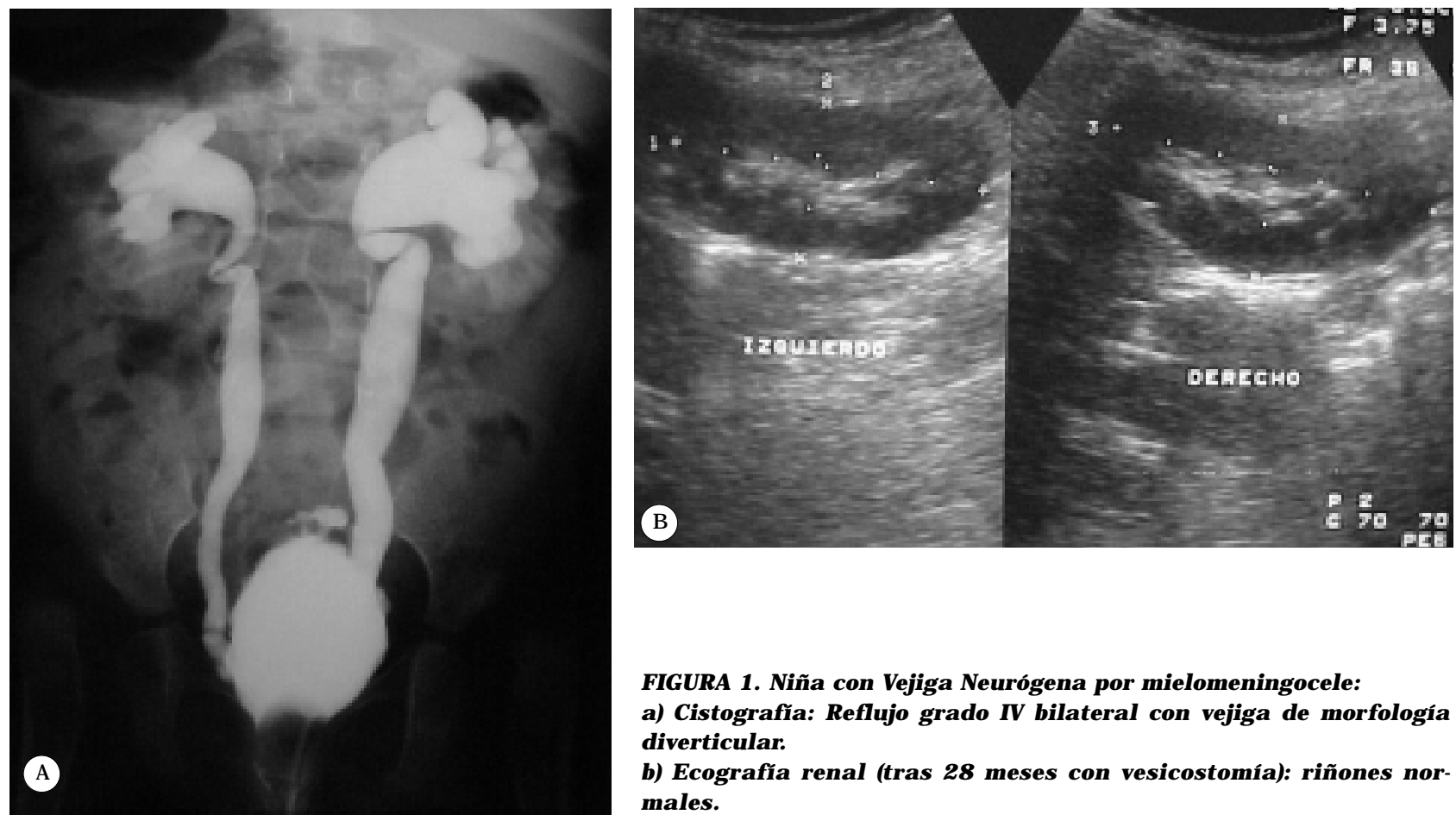

FIGURA 1. Niña con Vejiga Neurógena por mielomeningocele: a) Cistografia: Reflujo grado IV bilateral con vejiga de morfologia diverticular.

b) Ecografia renal (tras 28 meses con vesicostomia): riñones normales.

El cierre de la vesicostomía se ha realizado tras un tiempo medio de 3,2 años. El RVU desapareció en 5 pacientes, y sólo en 2 fue necesario realizar técnica antireflujo en el mismo acto quirúrgico.

En ningún caso ha habido recidiva del RVU, ectasia del tracto urinario superior, ni alteración de la dinámica miccional.

Seis niños afectos por Válvulas de Uretra Posterior fueron vesicostomizados, todos con UHN, reflujo vesicoureteral y afectación importante de la función renal. La edad media fue de 1,5 meses (rango: 1-2 meses). La UHN mejoró en el 100\% de los casos, desapareciendo el RVU en 50\% (Fig. 2). La vesicostomía se ha cerrado en todos ellos tras un periodo de tiempo medio de 4,7 años, requiriendo 2 pacientes ampliación vesical, para aumentar la capacidad.

De los 6 casos, 3 mantienen buena función renal, en 2 se ha realizado un trasplante y 1 permanece en diálisis, pendiente de injerto renal.

Las complicaciones se presentaron en 9 de los 43 casos (21\%) (Tabla II). Durante el primer año de seguimiento, apareció infecciones urinarias sintomáticas en 4 pacientes, desapareciendo posteriormente; la bacteriuria asintomática no se trató, a menos que el $\mathrm{Ph}$ urinario fuese elevado.
Prolapso vesical se produjo en 4 casos, requiriendo sólo una intervención quirúrgica para fijar la cúpula vesical. Litiasis vesical apareció en 3 pacientes y estenosis del estoma en 2 (que requirieron intervención quirúrgica correctora). No ha habido reacciones cutáneas importantes, secundarias al contacto de la orina con la piel.

\section{DISCUSIÓN}

La vesicostomía cutánea sigue siendo la derivación urinaria temporal no intubada más utilizada en la actualidad, aunque, son menos los niños que la precisan para mantener su tracto urinario estable. Pero la inquietud del urólogo frente a estos pacientes, debe ser la elección del tratamiento que mejor preserve la función renal, aunque sea a expensas de una derivación urinaria temporal.

Está indicada en los casos de obstrucción vesical, infravesical, UHN no obstructiva, con alteración de la función renal y/o infección urinaria persistente, en niños afectos de vejiga neuropática, reflujo vesicoureteral, válvulas de uretra posterior y Síndrome de Prune-Belly, que no responden a las medidas terapéuticas conservadoras. 

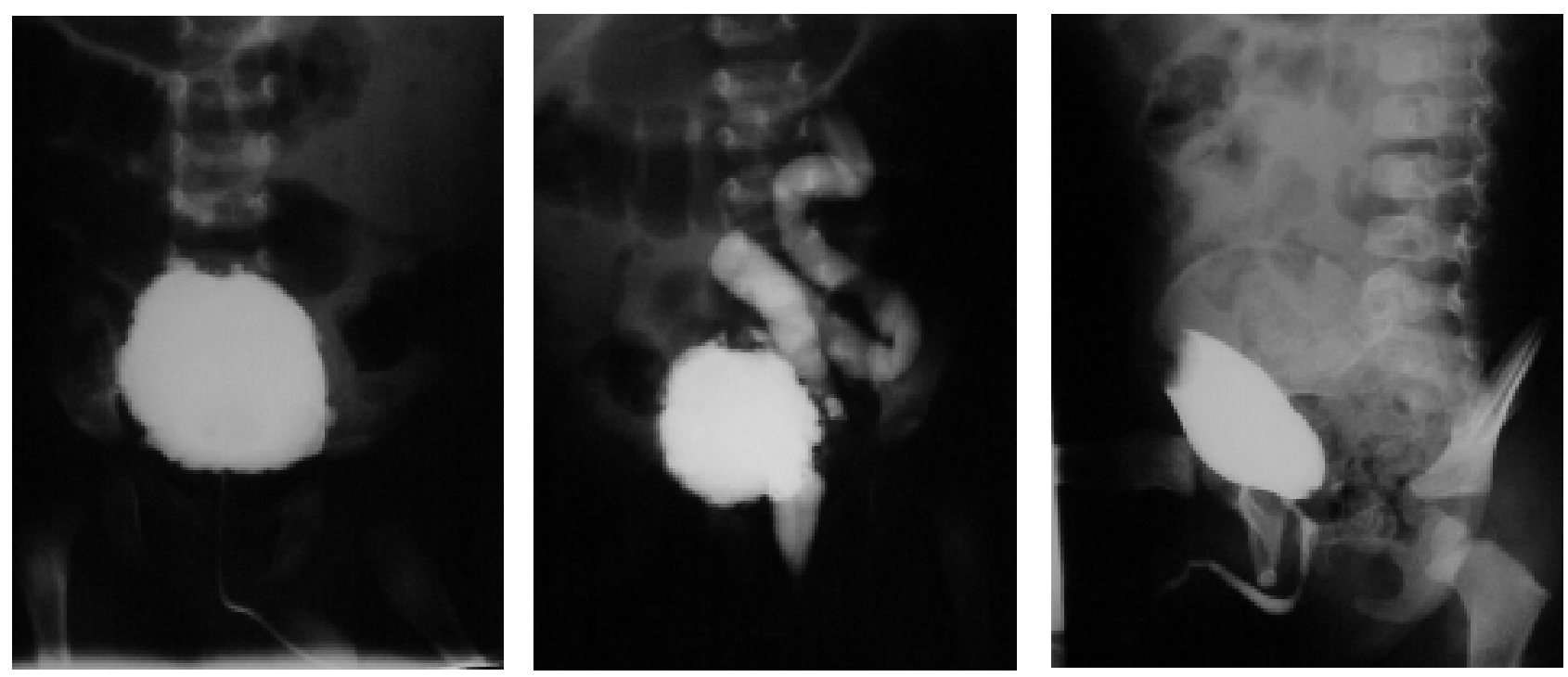

FIGURA 2. Niño afecto de Válvula de Uretra Posterior:

a) CUMS, fase de llenado: Vejiga diverticular.

b) CUMS, fase miccional: Reflujo Vesicoureteral grado 5. Uretra posterior dilatada.

c) Cistografía por vesicostomia (2 años después). Recuperación morfológica de la vejiga y uretra y desaparición del reflujo.

\section{TABLA II}

COMPLICACIONES DE LA VESICOSTOMÍA

\begin{tabular}{|l|c|c|c|}
\hline & VN & RVUP & VUP \\
\hline IUS & 1 & 2 & 1 \\
\hline Prolapso & 3 & 1 & - \\
\hline Litiasis & 2 & 1 & - \\
\hline Estenosis & 2 & - & - \\
\hline
\end{tabular}

VN: Vejiga neurógena

VUP: Válvulas de uretra posterior

RVUP: Reflujo vesicoureteral primario

IUS: Infección urinaria sintomática

Es una técnica de fácil realización, que proporciona una recuperación morfológica del tracto urinario, con baja incidencia de complicaciones.

El manejo de los pacientes con afectación neurogénica vesical es complejo, dependiendo de diversos factores: edad, sexo, tipo de disfunción vesical, malformaciones asociadas y ambiente y predisposición familiar para realizar el tratamiento indicado. La existencia de altas presiones intravesicales, condiciona con frecuencia la aparición de un reflujo vesicoureteral secundario ${ }^{3,4}$, que se incrementa con la edad y que condiciona la evolución de estos pacientes ${ }^{5,6}$. Las posibilidades de tratamiento son:
1. Tratamiento conservador, basado en programas de cateterismo intermitente, tratamiento farmacológico de la disfunción vesical y profilaxis antimicrobiana.

2. Derivación urinaria temporal.

3. Ampliación vesical.

4. Tratamiento precoz (endoscópico).

La corrección quirúrgica mediante cirugía abierta primaria, conlleva mayor morbilidad y peores resultados a largo plazo, frente a la derivación urinaria. Nosotros pensamos al igual que otros autores $^{3,7-9}$ que en los lactantes con mielodisplasia, la cateterización aséptica intermitente los tres primeros meses de vida, bajo profilaxis antimicrobiana, es la primera alternativa a considerar, y posteriormente en los casos de persistir RVU, asociaremos tratamiento farmacológico, para su disfunción vesical. La vesicostomía estaría indicada cuando a pesar de estas medidas, se presente daño renal y/o persistencia de infección urinaria.

Son muchas las publicaciones que han reportado excelentes resultados y escasas complicaciones. H. Snyder y col. ${ }^{10}$ en una serie de 48 niños con espina bífida y ureterohidronefrosis sometidos a vesicostomía, consigue la estabilización del TUS en 43 de 44 pacientes, persistiendo sólo en 3 casos la infección urinaria; las complicaciones fueron escasas: litiasis vesical en 3 niños y nece- 
sidad de revisión quirúrgica de la ostomía por estenosis o prolapso, en otros 4 niños. Krahn y col. ${ }^{8}$ en una revisión de 50 niños derivados por esta técnica (36 con RVU secundario), sólo necesitó aplicar una técnica antirreflujo en 5 de 26 casos, cuando se procedió al cierre de la vesicostomía y sólo 5 niños presentaron infecciones urinarias sintomáticas, 6 prolapso y 4 estenosis de la ostomía.

En la serie de S.K. Agarwal y col. ${ }^{3}$, sobre 319 niños con mielodisplasia, se analizan 94 casos con RVU asociado; de éstos, el 64\% (60 casos) se resolvieron de forma conservadora con cateterismo intermitente, antimicrobianos y anticolinérgicos, requiriendo el 23\% (22 niños), la realización de una vesicostomía, bien por persistencia del reflujo de alto grado o por deterioro del TUS; en el resto, se realizó directamente cirugía antirreflujo y/o cistoplastia de aumento. En los casos sometidos a la derivación, el reflujo se resolvió en 11 pacientes, requiriendo cirugía para su corrección unido o no a ampliación vesical en otros 11 . Por tanto, en el $76 \%$ de los niños, el RVU se resolvió sin necesidad de ningún procedimiento quirúrgico añadido.

En nuestra serie esta derivación ha permitido resolver definitivamente el reflujo en el $65 \%$ de casos y el restablecimiento de una función renal normal en el $90 \%$. La UHN mejoró en el 100\% y las infecciones urinarias sintomáticas desaparecieron tras el primer año de seguimiento.

En los neonatos con Válvulas de Uretra Posterior ha disminuido la indicación de derivación, realizándose en estos pacientes el tratamiento endoscópico, tras el diagnóstico y mejora del estado general y metabólico.

En los inicios de la década de los 80, Krueger y col. ${ }^{11}$, afirmaron que aquellos niños con derivación transitoria, se desarrollaban somáticamente mejor y con tasas menores de creatinina en suero, que los tratados mediante ablación primaria. Sin embargo otros autores ${ }^{12}$ no encontraron diferencia entre el manejo inicial con vesicostomía o resección endoscópica. Reinberg y col. ${ }^{13}$ demostraron que el tratamiento inicial no influía en la progresión del daño renal y crecimiento del niño. Pero la tendencia actual es a la ablación primaria, pues, aunque no hay diferencias en cuanto a la función renal, los pacientes presentan mejor funcionalismo vesical ${ }^{14} \mathrm{y}$ menor número de intervenciones posteriores ${ }^{15}$.
En caso de realizarse una derivación, Jaureguizar y col. ${ }^{16}$ consideran la ureterostomía bilateral sistemática como mejor opción frente a la vesicostomía, ya que en casos severos puede mejorar la función renal, sin incrementar la disfunción vesical.

La aparición de alteraciones del funcionalismo vesical, secundarias a la derivación, no han sido demostradas, poniéndose de manifiesto la ausencia de diferencias entre pacientes sometidos a derivación tipo ureterostomía, vesicostomía o ablación primaria ${ }^{14,16}$. Para Lal R. y col. ${ }^{17}$, la alteración de la capacidad, "compliance" y contractilidad vesical en estos niños, aparece en ambas modalidades de tratamiento, ya que serian debidas a alteraciones congénitas, inherentes al desarrollo del detrusor.

En nuestra serie la vesicostomía permitió el restablecimiento de la función renal en 3 de los 6 pacientes con VUP, sin apreciar alteraciones urodinámicas importantes, salvo dos pacientes con leve alteración del vaciado (uno de ellos sometido a ampliación vesical). Nosotros consideramos que la derivación (ureterostomía o pielostomía) debe reservarse para casos en los que tras el tratamiento endoscópico o la derivación temporal (cateterismo uretrovesical o cistostomía suprapúbica) no se logra mejorar el estado metabólico o disminuir la dilatación del TUS.

Pensamos que los niños afectos de uropatía secundaria a obstrucción del tramo común con descenso de los niveles de creatinina sérica, mejoría de la ureteroectasia y sin reflujo o con mejoría tras la descompresión, serían susceptibles de ablación primaria y los casos con un reflujo vesicoureteral severo, insuficiencia renal (Creatinina $>1,6 \mathrm{mgr} / \mathrm{dl}$ ) o alteración del estado general serían los candidatos para la realización de la derivación. Igualmente estaría indicada en neonatos o lactantes pequeños con RVU de alto grado, en los que a pesar de la profilaxis antibiótica su evolución es tórpida, con infecciones urinarias persistentes y consiguiente daño renal.

Las complicaciones más frecuentes de esta derivación son la infección urinaria persistente y/o recidivante, la estenosis del estoma, litiasis y prolapso vesical ${ }^{1,9,18}$. La bacteriuria suele ser constante, pero sólo en los casos de producir sintomatología y la posibilidad de litiasis (ph urinario elevado) es necesario su tratamiento. 
El prolapso es la complicación más habitual, debida a una técnica no adecuada (más frecuente cuando se realiza el procedimiento de Lapides), y suele asociarse a incrementos de la presión intraabdominal, manejándose normalmente con reducción manual. C.C. Chu y col. ${ }^{19}$ describen un caso de prolapso vesical junto a estrangulación de asas intestinales. Entre las complicaciones anecdóticas está la aparición de neoplasias ${ }^{20}$, por lo que Sonda L.P. y col. recomiendan biopsia de aquellas lesiones que resulten sospechosas.

En la serie de Noe y R. Jerkins ${ }^{18}$, sobre un total 35 niños con vesicostomía, aparecieron complicaciones en 14 pacientes (infecciones urinarias importantes en 6 y en otros 6 prolapso vesical), y sólo requirieron cirugía dos de ellos, uno por estenosis del estoma y otro por prolapso vesical masivo. La dermatitis periestomal significativa apareció sólo en un niño.

La complicación más seria, pero muy poco frecuente, es el fallo en la descompresión del tracto urinario superior, con el consiguiente agravamiento del daño renal. Nuestra serie presenta también escasa incidencia de complicaciones importantes, ya que sólo 3 pacientes requirieron intervención quirúrgica ( 2 por estenosis del estoma y 1 por prolapso vesical masivo).

\section{CONCLUSIONES}

Consideramos que la vesicostomía cutánea es una opción válida, en niños de la primera infancia, que presentan una uropatía obstructiva vesical, infravesical o UHN no obstructiva, que con otras medidas terapéuticas no consiguen mejorar el funcionalismo renal ó la desaparición de las infecciones urinarias. El cierre de la misma debe realizarse tan pronto como sea posible, una vez corregida su patología base o reconstruido el tracto urinario mediante otras técnicas quirúrgicas.

\section{REFERENCIAS}

1. DIXON WALQUER R.: ¿Quién necesita derivación urinaria transitoria?. En: Cirugia Urológica Pediátrica. Ed. Interamericana 1988: 229-240.

2. DUCKETT JW.: Cutaneous vesicostomy in childhood: the Blocksom technique. Urol Clin North Am 1974; 1: 485495.

3. AGARWAL SK, KHOURY AE, ABRAMSON RP, CHURCHILL BM, ARGIROPOULOS G, McLORIE GA.: Outcome analysis of vesicoureteral reflux in children with myelodysplasia. J Urol 1997; 157: 980-982.
4. KOFF SA.: Relationship between dysfunctional voiding and reflux. $J$ Urol 1992; 148: 1.703-1.705.

5. COHEN RA, RUSHTON HG, BELMAN AB, KASS EJ, MAJD M, SHAER C.: Renal scarring and vesicoureteral reflux in children with myelodysplasia. J Urol 1990; 144: 541-544.

6. KAPLAN WE, FIRLIT CF.: Management of reflux in the myelodysplastic child. J Urol 1983; 129: 1.195-1.197.

7. KLOSE AG, SACKETT CK, MESROBIAN HJ.: Management of children with myelodysplasia: urological alternatives. $J$ Urol 1990; 144: 1.446-1.449.

8. KRAHN CG, JOHNSON HW.: Cutaneous vesicostomy in the young child: indications and results. Urology 1993; 41: 558-563.

9. GARCÍA MÉRIDA M, MIGUÉLEZ LAGO C, MARQUÉS GUBERN A, GARCÍA ROMERO J, LÓPEZ MARTÍNEZ A, ISNART R.: El primer año de la vida del niño con mielodisplasia. Estudio multicéntrico sobre 393 casos. Cir Pediatr 1996; 9: 3-9.

10. SNYDER H, KALICHMAN MA, CHARNEY E, DUCKETT JW.: Vesicostomy for neurogenic bladder with spina bifida: followup. J Urol 1983; 130: 724-726.

11. KRUEGER RP, HARDY BC, CHURCHILL BM.: Growth in boys with posterior urethral valves. Urol Clin North Am 1980; 7: 265-272.

12. DIXON WALQUER R, PADRON M.: The management of posterior urethral valves by initial vesicostomy and delayed valve ablation. $J$ Urol 1990; 144: 1.212-1.214.

13. REINBERG Y, DE CASTANO I, GONZÁLEZ R.: Influence of initial therapy on progression of renal failure and body growth in children with posterior urethral valves. J Urol 1992; 148: 532-533.

14. PODESTA ML, RUARTE A, GARGIULO C, MEDEL R, CASTERA R.: Urodynamic findings in boys with posterior urethral valves after treatment with primary valve ablation or vesicostomy and delayed ablation. $J$ Urol 2000; 164: 139-144.

15. SMITH GRAHAME HH, CANNING DA, SCHULMAN SL, SNYDER HM, DUCKETT JW.: The long-term outcome of posterior urethral valves treated with primary valve ablation and observation. J Urol 1996; 155: 1.7301.734 .

16. JAUREGUIZAR E, LÓPEZ PEREIRA P, MARTÍNEZ URRUTIA MJ, ESPINOSA L.: Does neonatal pyeloureterostomy worsen bladder function in children with posterior urethral valves?. J Urol 2000; 164: 1.031-1.033.

17. LAL R, BHATNAGAR LR, AGARWALA S, GROVER VP, MITRA DK.: Urodynamic evaluation in boys treated for posterior urethral valves. Pediatr Surg Int 1999; 15: 358-362.

18. NOE HN, JERKINS GR.: Cutaneous vesicostomy experience in infants and children. J Urol 1985; 134: 301303.

19. CHIH-CHUN C, GUAN-YEU D.: Prolapsed vesicostomy results in a strangulated bowel herniation: a rare complication of cutaneous vesicostomy. J Urol 1994; 152: $1.572-1.573$.

20. SONDA LP, SOLOMON MH.: Twenty-year outcome of cutaneous vesicostomy. J Urol 1980; 124: 326-328.

\footnotetext{
Dr. J.A. Queipo Zaragozá

Calle Santa Teresa, 4-8

46530 Puzol (Valencia)
}

(Trabajo recibido el 11 junio de 2002) 